\title{
Optimum short-time polynomial regression for signal analysis
}

\author{
A SREENIVASA MURTHY ${ }^{1}$, CHANDRA SEKHAR SEELAMANTULA $^{2, *}$ and T V SREENIVAS ${ }^{3}$ \\ ${ }^{1}$ Department of Electronics and Communication Engineering, University Visveswaraya College of Engineering, \\ Bangalore University, Bangalore 560001, India \\ 2 Department of Electrical Engineering, Indian Institute of Science, Bangalore 560012, India \\ ${ }^{3}$ Department of Electrical Communication Engineering, Indian Institute of Science, Bangalore 560012, India \\ e-mail: uvceasm@gmail.com; chandra.sekhar@ieee.org; tvsree@ece.iisc.ernet.in
}

MS received 3 October 2011; revised 7 May 2016; accepted 10 June 2016

\begin{abstract}
We propose a short-time polynomial regression (STPR) for time-varying signal analysis. The advantage of using polynomials is that the notion of a spectrum is not needed and the signals can be analyzed in the time domain over short durations. In the presence of noise, such modeling becomes important, because the polynomial approximation performs smoothing leading to noise suppression. The problem of optimal smoothing depends on the duration over which a fixed-order polynomial regression is performed. Considering the STPR of a noisy signal, we derive the optimal smoothing window by minimizing the mean-square error (MSE). For a fixed polynomial order, the smoothing window duration depends on the rate of signal variation, which, in turn, depends on its derivatives. Since the derivatives are not available a priori, exact optimization is not feasible. However, approximate optimization can be achieved using only the variance expressions and the intersection-ofconfidence-intervals (ICI) technique. The ICI technique is based on a consistency measure across confidence intervals corresponding to different window lengths. An approximate asymptotic analysis to determine the optimal confidence interval width shows that the asymptotic expressions are the same irrespective of whether one starts with a uniform sampling grid or a nonuniform one. Simulation results on sinusoids, chirps, and electrocardiogram (ECG) signals, and comparisons with standard wavelet denoising techniques, show that the proposed method is robust particularly in the low signal-to-noise ratio regime.
\end{abstract}

Keywords. Short-time polynomial regression (STPR); mean-square error; bias-variance trade-off; confidence interval; intersection of confidence intervals (ICI) technique.

\section{Introduction}

Most real-world signals are time-varying, and the notion of a global Fourier spectrum has limited applicability in such cases. Real-world signals are also smooth and amenable to local approximation by polynomials. The advantage of a local model is that it gives flexibility in terms of adapting the model to the temporal signal properties. Part of the motivation for the short-time polynomial model also lies in the Taylor series representation, according to which smooth functions can be expressed point-wise as polynomials of infinite degree, whose coefficients are given by the derivatives of the function at that point. Another relevant result is the Weierstrass approximation theorem [1] according to which a smooth function can be approximated to a desired

\section{*For correspondence}

Preliminary results reported in this paper were published in the Proceedings of European Signal Processing Conference (EUSIPCO) 2008. The results reported in this paper are based on the Ph.D. thesis of the first author awarded by IISc in 2013. accuracy over an interval using polynomials. The difference in the two approaches is that the Taylor series representation is a point-wise polynomial approximation, whereas the Weierstrass theorem deals with interval-based approximations. In this paper, we consider polynomial approximations on intervals. Whereas in Weierstrass' formulation, the fixed parameters are the interval width and the accuracy of fit, and the degree of the polynomial is the free parameter, in our formulation, the optimal degree of the polynomial is determined based on experimental analysis and the optimal duration is adjusted at every instant.

The short-time polynomial model has the advantage that it does not require transformation to a different domain (frequency domain, for example). Model-fitting is done by working in the time domain. This feature allows the model to adapt to the time-varying behavior of the signal and does not require the assumption of bandlimitedness. In fact, strictly speaking, most practical signals are not bandlimited. An aspect that requires consideration is the duration over which the regression is performed and the order of the polynomial. In the presence of measurement noise, this issue becomes 
important because it introduces a trade-off between the amount of smoothing and the accuracy of fit. Typically, for large windows, we get smooth estimates (small variance), but the deviation from the ground truth is large (large bias). The converse happens for short windows. Therefore, the window duration has to be adapted instantaneously to obtain best regression results - investigating this aspect for both uniform and nonuniform sampling is the focus of our paper.

Short-time polynomial modeling is widely used for kernel density estimation $[2,3]$. In the discrete setting, it has been used to perform data smoothing. In a seminal paper, Savitzky and Golay [4] showed that short-time polynomial modeling is equivalent to discrete-time convolution with a fixed filter that depends on the window size and order of the polynomial. In an expository article, Schafer illustrated several interesting properties of the Savitzky-Golay (SG) filters [5]. Krishnan and Seelamantula [6] used the concept of risk minimization to determine the optimal window length and order parameters of the SG filters. In this paper, we take a sampling-theoretic perspective of reconstructing a continuous-domain signal from its samples (uniform grid or nonuniform grid) and show that there is a trade-off between the adaptability of the model to local signal variations and robustness to noise, which is equivalent to the bias-variance trade-off [7]. We consider two scenarios: (i) uniformly sampled signals and (ii) nonuniformly sampled signals. In both cases, we derive the bias-variance trade-off expressions and show that, asymptotically, as the sampling becomes finer, both scenarios have identical bias-variance trade-off characteristics. We solve the trade-off using the intersection of confidence intervals (ICI) technique introduced by Katkovnik and Stankovic in the context of instantaneous frequency estimation [8,9]. The short-time polynomial regression (STPR) approach has received a lot of attention for biomedical applications. For example, Zhang et al [10] used the short-time polynomial model for time-frequency analysis of event-related electroencephalograph (EEG) signals within the framework of autoregressive modeling. In this paper, we promote STPR analysis and the ICI technique, and show some applications to ECG signal denoising, nonuniform signal reconstruction from noisy samples, and denoising of chirp signals.

On the nonuniform sampling front, an early seminal work is that of Yao and Thomas [11], who stated that bandlimited signals can be uniquely determined from nonuniform samples provided that the average sampling rate exceeds the Nyquist rate. A review of some reconstruction methods can be found in the tutorial by Jerri [12]. Over the past few decades, several variants of nonuniform sampling and reconstruction have been proposed. Efficient filterbank-based reconstruction techniques for signals from recurrent nonuniform samples was developed in [13]. Practical finiteimpulse-response (FIR) filterbank-based reconstruction of nonperiodic-bandlimited signals from recurrent nonuniform samples was developed by Prendergast et al [14]. The reconstruction is not exact but optimal in the least-squares and minimax sense. The problem of recovering periodic bandlimited signals based on nonuniform samples was addressed by Margolis and Eldar [15], who provided closedform solutions in the cases of bases as well as frames. Strohmer and Tanner [16] proposed fast reconstruction algorithms for periodic, nonuniformly spaced samples, where the sampling rate is at least the Nyquist rate. They also provided applications to time-interleaved analog-to-digital converters (ADCs). Strohmer also developed a fast multilevel algorithm for signal reconstruction from irregular samples in trigonometric bases. Divi and Wornell [17] addressed a similar problem in the context of periodic nonuniform samples and provided a constrained leastsquares estimate of the signal based on a time-varying filter. The locations of the irregular set of samples are determined using random search and cyclic coordinate methods. Wavelet-type solutions were presented for the interpolation and denoising of nonuniformly sampled signals [18].

The papers cited so far assume either a bandlimited or a periodic signal. Our model is based on short-time polynomial modeling and does not make such assumptions. Laakso et al [19] provided a reconstruction algorithm using time-varying FIR filters on the assumption that the signal is locally smooth. However, their reconstruction is not optimal in the MMSE sense.

\subsection{Organization of the paper}

In section 2, we formulate short-time polynomial regression (STPR) for uniformly sampled signals. Based on an example, we motivate the need for adapting the window as a function of time. The bias and variance have complementary characteristics as a function of the window length. In section 3, the nonuniform sampling counterpart of the problem addressed in section 2 is taken up. We show that there exist similar tradeoffs in the nonuniform scenario as well. Further, the window length can be optimized to resolve the bias-variance trade-off, using the ICI technique, which has been used in other estimation problems. A brief outline of the ICI technique and a modified cost function to determine its parameters is given in section 4 . In section 5 contains the experimental results related to the uniform sampling scenario, signal reconstruction from nonuniform samples, denoising of chirp signals based on STPR, denoising of ECG signals, and performance comparison with wavelet denoising techniques.

\subsection{Contributions}

The key contributions of this paper are: (i) A unified short-time polynomial modeling approach for uniformly or nonuniformly sampled signals and derivation of the associated bias-variance trade-off expressions; (ii) Solving the bias-variance trade-off using the ICI technique; (iii) Reliable determination of ICI parameters; and (iv) Experimental validation on synthesized chirp signals, real ECG data, and comparisons with some standard wavelet denoising techniques. 


\section{Short-time polynomial modeling - uniform sampling scenario}

Let $s[n T]$ denote the samples ( $T$ denotes the sampling period) of a signal $s(t)$ corrupted with additive white Gaussian noise samples $w[n T] \sim \mathcal{N}\left(0, \sigma_{w}^{2}\right)$, to yield the noisy measurements:

$$
x[n T]=s[n T]+w[n T], \quad 0 \leq n \leq N-1 .
$$

The objective is to obtain an estimate of $s(t)$ at an instant $n T$ by performing short-time polynomial regression (STPR) of a certain order $p$, over the interval $\left[n T-\frac{L}{2}, n T+\frac{L}{2}\right]$ as

$$
s[n T]=\sum_{k=0}^{p} a_{k}(n T)^{k}+\Delta s[n T]
$$

where the parameter vector $\theta=\left[a_{0}, a_{1}, a_{2}, \ldots, a_{p}\right]$ has to be estimated and $\Delta s[n T]$ is the modeling error between the signal $s[n T]$ and its finite-order short-time polynomial approximation $\sum_{k=0}^{p} a_{k}(n T)^{k}$. In terms of the polynomial model for $s[n T]$, the noisy signal $x[n T]$ assumes the form:

$$
x[n T]=\sum_{k=0}^{p} a_{k}(n T)^{k}+\Delta s[n T]+w[n T] .
$$

Let $\widehat{s}[n T]$ denote an estimator of $s[n T]$. Given $x[n T]$, the objective is to compute $\widehat{s}[n T]$ such that the mean-square error (MSE) $=\mathcal{E}\left\{(s[n T]-\widehat{s}[n T])^{2}\right\}$ is minimized, that is, we would like to compute the MMSE estimator of $s[n T]$. We shall show that the MSE is a function of the window length $L$. Hence, given a polynomial of a certain order, and knowledge about noise statistics (mean, variance, and Gaussian-ness of the noise), the problem reduces to finding the value of $L$ that yields the minimum MSE.

\subsection{Fixed-window polynomial regression}

In order to demonstrate the importance of the window length over which the short-time polynomial modeling is performed, we synthesize a signal containing different types of sub-signals: a short sinusoidal segment, followed by a rectangular pulse, a decaying exponential, a parabola and a decaying sinusoid, with zeros inserted between the sub-signals. The synthesized signal is shown in figure 1(a). A noisy version of the signal is generated by adding pseudo-random white noise sequence to the synthesized signal (cf. figure 1(b)). The signal-to-noise ratio (SNR) is maintained at $10 \mathrm{~dB}$. The SNR is computed as the ratio of sample variances of the noise-free signal to that of noise. We then perform STPR at every point of the noisy signal over a local neighborhood. The order of the polynomial is fixed as three and the results of polynomial fitting, corresponding to two different window lengths (short window: 9 samples and long window: 79 samples) are shown in figure 1(c) and (d), respectively. By performing STPR over long durations, one can achieve smooth estimates of the underlying signal. The problem, however, lies in the
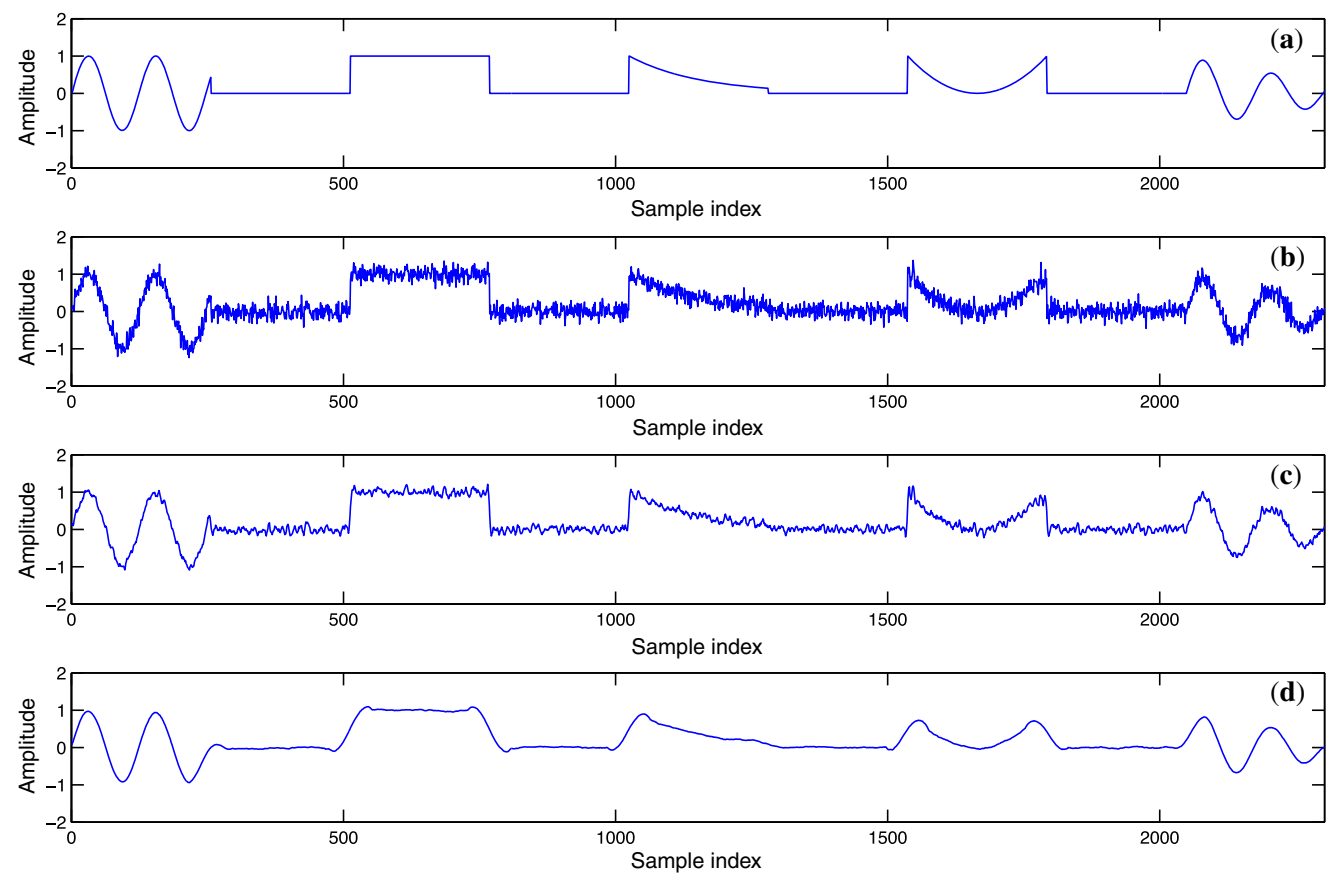

Figure 1. (a) Noise-free signal, (b) noisy signal $(\mathrm{SNR}=10 \mathrm{~dB})$, enhanced signal using a third-order STPR at every point, by taking a (c) 9-point window, and (d) 79-point window. 
transition regions. The transitions are smeared by the longduration smoothing (less variance, but more bias). On the other hand, short-time polynomial smoothing over short durations has the advantage that it preserves the transitions accurately, but it does not average out noise well in the steady regions (less bias, but more variance). Ideally, one needs a technique that can adapt the duration of the STPR based on the local signal properties. In other words, one has to adaptively trade-off between the bias and variance of the estimator in order to achieve an optimal fit in terms of the mean-square error. The problem appears somewhat complicated mainly because the window length must be chosen to adapt to the underlying noise-free signal properties, but the signal itself is not known a priori. Our objective is to develop a practical approach that enables optimal choice of the window duration at every instant.

\subsection{Bias and variance calculations}

Consider the polynomial regression of $x[m T]$. Let $\mathcal{C}(m T, \theta)$ denote the regression error:

$$
\mathcal{C}(m T, \theta)=\sum_{n T=m T-\frac{L}{2}}^{m T+\frac{L}{2}}\left(s[n T]+w[n T]-\sum_{k=0}^{p} a_{k}(n T)^{k}\right)^{2} h(m T-n T),
$$

where $h(t)$ is a real-valued window function of length $L$ and symmetric about $t=0$. The role of $h(t)$ is to localize the polynomial fitting about the instant $m T$, and also to weigh the modeling errors. The optimum coefficient estimates, $\widehat{a}_{\ell}, 0 \leq \ell \leq p$ are obtained as

$$
\widehat{a}_{\ell}=\arg \min _{a_{\ell}} \mathcal{C}, 0 \leq \ell \leq p .
$$

Differentiating $\mathcal{C}$ with respect to $a_{\ell}$, we get

$$
\begin{aligned}
& \frac{\partial \mathcal{C}}{\partial a_{\ell}}=-2 \sum_{n T=m T-\frac{L}{2}}^{m T+\frac{L}{2}}\left(s[n T]+w[n T]-\sum_{k=0}^{p} a_{k}(n T)^{k}\right)(n T)^{\ell} h(m T-n T), \\
& 0 \leq \ell \leq p \text {. }
\end{aligned}
$$

In order to assess the relative contributions of the model mismatch and the additive noise to the estimation error, the above expression is rearranged as

$$
\frac{\partial \mathcal{C}}{\partial a_{\ell}}=\left.\frac{\partial \mathcal{C}}{\partial a_{\ell}}\right|_{0}+\left.\frac{\partial^{2} \mathcal{C}}{\partial a_{\ell}^{2}}\right|_{0} \Delta a_{\ell}+\left.\frac{\partial \mathcal{C}}{\partial a_{\ell}}\right|_{0} \delta_{\Delta_{s}}+\left.\frac{\partial \mathcal{C}}{\partial a_{\ell}}\right|_{0} \delta_{w},
$$

where

- $\Delta a_{\ell}=a_{\ell}-\widehat{a}_{\ell}$ is the estimation error due to modeling inaccuracy and the effect of noise;

- $\left.\frac{\partial \mathcal{C}}{\partial a_{\ell}}\right|_{0}$ is the derivative of $\mathcal{C}$ with respect to $a_{\ell}$ in the absence of noise and mismatch errors (indicated by $\left.\right|_{0}$ );
- $\left.\frac{\partial^{2} \mathcal{C}}{\partial a_{\ell}^{2}}\right|_{0} \Delta a_{\ell}$ is the perturbation in $\frac{\partial \mathcal{C}}{\partial a_{\ell}}$ due to estimation error in $a_{\ell}$;

- $\left.\frac{\partial \mathcal{C}}{\partial a_{\ell}}\right|_{0} \delta_{\Delta_{s}}$ is the perturbation in $\frac{\partial \mathcal{C}}{\partial a_{\ell}}$ due to model mismatch; and

- $\left.\frac{\partial \mathcal{C}}{\partial a_{\ell}}\right|_{0} \delta_{w}$ is the perturbation in $\frac{\partial \mathcal{C}}{\partial a_{\ell}}$ due to additive noise.

The evaluation of each of the above quantities, and the subsequent calculations and simplifications (using the idea of data centering), which lead to the bias and variance, is given in Appendix A. We recall here the final expressions for the bias and variance:

$$
\mathcal{E}\{\Delta s(0)\}= \begin{cases}\frac{s^{(p+1)}(0)}{(p+2) !}\left(\frac{L}{2}\right)^{p+1} & p \text { odd }, \\ \frac{s^{(p+2)}(0)}{(p+3) !}\left(\frac{L}{2}\right)^{p+2} & p \text { even },\end{cases}
$$

and $\operatorname{Var}(\Delta s(0))=\operatorname{Var}\left(\Delta a_{0}\right)=\frac{\sigma_{w}^{2}}{L}$.

The squared bias is proportional to $L^{2 p+2}$ or $L^{2 p+4}$, according as $p$ is odd or even, respectively. On the other hand, the variance is inversely proportional to $L$. The complementary dependence of bias and variance on $L$ explains the bias-variance trade-off, which is a central concept in parameter/signal estimation using finite-length data [7,9]. The increased smoothing by increasing window length decreases variance but increases bias. Reduced smoothing for decreasing window lengths increases variance

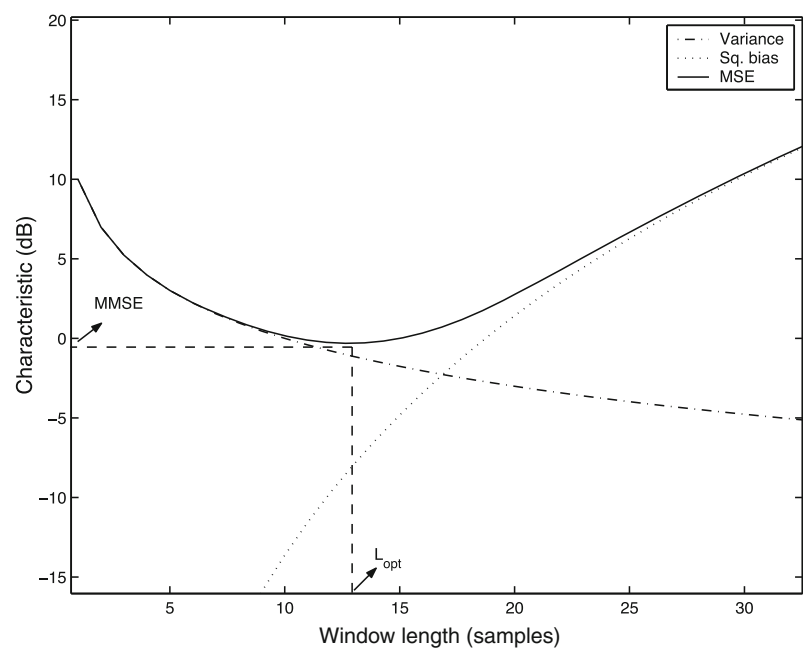

Figure 2. Illustration of bias-variance trade-off. The $y$-axis is labeled as 'Characteristic' as a common reference to squared bias, variance, and MSE. 


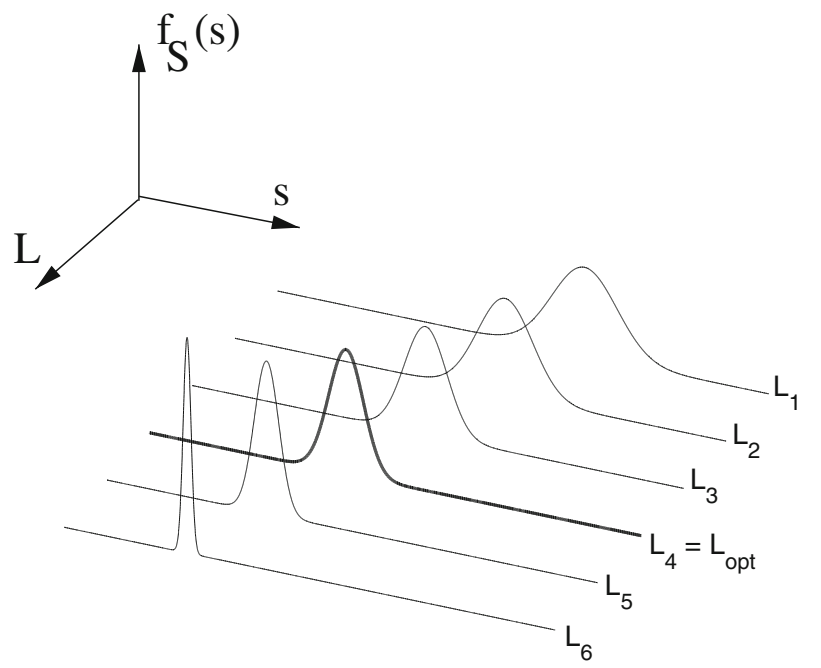

Figure 3. Distribution of the estimator corresponding to different window lengths.

but decreases bias. Figure 2 shows a plot of the bias, variance, and mean-square error (MSE $=\mathrm{Bias}^{2}+$ Variance $)$ as a function of the window length for a specific example obtained using the expressions derived above. The parameters have been chosen arbitrarily. Bias and variance have complementary behavior as a function of window length, and hence, it is not possible to simultaneously minimize both of them.

In terms of the distribution of the estimator, the tradeoff implies that as the distribution tends to be centered (corresponding to small window lengths) about the ground truth value of the parameter being estimated, the spread of the distribution increases. On the other hand, as the distribution tends to be centered away from the actual value (corresponding to large window lengths), the spread decreases. This behavior is illustrated in figure 3. The optimum window length estimator distribution offers the best combination in terms of the spread (standard deviation) and distance from the parameter being estimated (bias). In the sequel, without loss of generality, we assume that $p$ is odd.

\subsection{Optimum window length}

The expression for the MSE is

$$
\operatorname{MSE}(\widehat{s}(0))=\frac{\left[s^{p+1}(0)\right]^{2} L^{2 p+2}}{[(p+2) !]^{2} 2^{2 p+2}}+\frac{\sigma_{w}^{2}}{L} .
$$

The optimum window length $L_{\text {opt }}$ corresponds to minimum mean-square error. By minimizing (2) with respect to $L$, we get that $L_{\mathrm{opt}}=\left(\frac{\sigma_{\alpha^{2}}^{2} 2 p+2[(p+2) !]^{2}}{\left[s^{p+1}(0)\right]^{2}[(2 p+2)}\right)^{\frac{1}{2 p+3}}$.

For $L=L_{\mathrm{opt}}$,

$$
\begin{aligned}
(\mathcal{E}(\Delta s(0)))^{2} & =\frac{\mathcal{B}^{\frac{1}{2 p+3}} \sigma_{d}^{\frac{2(2 p+2)}{2 p+3}}}{(2 p+2)^{\frac{2 p+2}{2 p+3}}}, \\
\operatorname{Var}(\Delta s(0)) & =\sigma_{d}^{\frac{2(2 p+2)}{2 p+3}} \mathcal{B}^{\frac{1}{2 p+3}}(2 p+2)^{\frac{1}{2 p+3}}, \\
\mathcal{E}(\Delta s(0)) & =\sqrt{\frac{1}{2 p+2}} \sigma_{L_{\mathrm{opt}}},
\end{aligned}
$$

where

$$
\sigma_{L_{\mathrm{opt}}}=\left.\sigma_{L}\right|_{L=L_{\mathrm{opt}}}=\sqrt{\operatorname{Var}(\Delta s(0))}, \quad \text { and } \quad \mathcal{B}=\frac{\left[s^{p+1}(0)\right]^{2}}{[(p+2) !]^{2} 2^{2 p+2}} .
$$

The optimum window length is a function of the higherorder derivatives of the signal, which are not known a priori because the noise-free signal is not accessible. The expression for the optimum window length is insightful, but it has limited practical applicability. However, unlike the expression for bias, the expression for variance does not require prior knowledge of the signal. It depends only on the noise variance, which can be estimated accurately. The expression for variance can be used in the adaptive window algorithm to solve the bias-variance trade-off and achieve MMSE estimation. Assuming data centering, we drop the time index in $\Delta s(0)$ and $\widehat{s}(0)$. We use the notation $\widehat{s}_{L}$ to denote the estimate obtained using a rectangular window of length $L$.

\section{Short-time polynomial modeling - nonuniform sampling scenario}

Consider the practical sampling scenario as shown in figure 4 . The analog source output $s(t)$ is corrupted by measurement/observation noise $w(t)$. The samples of the process $y(t)=s(t)+w(t)$ are taken at ordered, nonuniform instants $\left\{t_{n}, t_{n}<t_{n+1}, n \in \mathbb{Z}\right\}$ resulting in the measurement

$$
y_{s}(t)=y(t) \sum_{n} \delta\left(t-t_{n}\right)=\sum_{n} y\left(t_{n}\right) \delta\left(t-t_{n}\right) .
$$

The noise $w(t)$ is assumed to be white Gaussian with zero mean and variance $\sigma_{w}^{2}$. The nonuniform sampling structure and noise are encountered in practical data acquisition scenarios such as observation data recorded in astronomy,

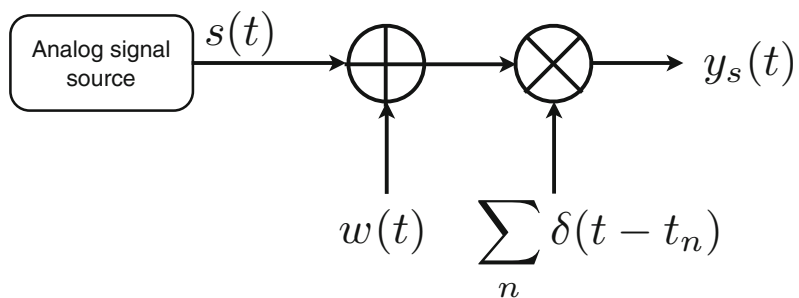

Figure 4. Schematic of a data-acquisition system with nonuniform sampling. 
data measured in a moving vehicle for oceanographic applications, magnetic/gravitational field measurements in geophysics, jitter in sampling, data loss in images or audio signals due to channel erasures, etc. [20, 21]. The observations are given by $y_{s}\left(t_{n}\right)=y\left(t_{n}\right)=s\left(t_{n}\right)+w\left(t_{n}\right)$. The sampling instants are assumed to be deterministic but nonuniformly spaced and unknown a priori. The goal is to obtain an estimate of $s(t)$ from the noisy measurements, $\left\{y\left(t_{n}\right), n \in \mathbb{Z}\right\}$ such that the MSE is least. The signal $s(t)$ is not constrained to be bandlimited.

\subsection{Bias and variance calculations}

Consider a positive-valued, symmetric window function $h(t)$ such that $\int_{-\infty}^{+\infty} h(t) \mathrm{d} t=1$ and $\int_{-\infty}^{+\infty} h^{2}(t) \mathrm{d} t<\infty$. Let $t$ be a point where the estimate of $s(t)$ is computed by a local $p^{\text {th }}$ order polynomial regression to the signal $s(t)$. Consider the cost function:

$$
\mathcal{C}(t, \mathbf{a})=\sum_{n}\left[y\left(t_{n}\right)-\sum_{k=0}^{p} a_{k}(t) t_{n}^{k}\right]^{2} h\left(t-t_{n}\right),
$$

where $\mathbf{a}=\left[a_{0}(t), a_{1}(t), a_{2}(t), \ldots, a_{p}(t)\right]$. The coefficients, $\left\{a_{k}(t), k=0,1,2, \ldots, p\right\}$ are functions of time. Denote the optimal coefficients by $\left\{\widehat{a}_{k}(t), k=0,1,2, \ldots, p\right\}$, obtained by minimizing $\mathcal{C}(t, \mathbf{a})$ with respect to $a_{\ell}(t), 0 \leq \ell \leq p$, that is, $\widehat{a}_{\ell}(t)=\arg \min _{a_{\ell}} \mathcal{C}(t, \mathbf{a})$. Setting the derivative to zero, that is, $\left.\frac{\partial \mathcal{C}}{\partial a_{\ell}(t)}\right|_{a_{\ell}(t)=\widehat{a}_{\ell}(t)}=0$, gives

$$
\begin{gathered}
\frac{\partial \mathcal{C}}{\partial a_{\ell}}=-2 \sum_{n}\left[s\left(t_{n}\right)+w\left(t_{n}\right)-\sum_{k=0}^{p} a_{k}(t) t_{n}^{k}\right] h\left(t-t_{n}\right) t_{n}^{\ell}, \\
\quad 0 \leq \ell \leq p .
\end{gathered}
$$

For brevity, the time-dependence of the polynomial coefficients is suppressed with the implicit understanding that they are time-varying. The derivative of the cost-function is split as follows:

$$
\frac{\partial \mathcal{C}}{\partial a_{\ell}}=\left.\frac{\partial \mathcal{C}}{\partial a_{\ell}}\right|_{0}+\left.\frac{\partial^{2} \mathcal{C}}{\partial a_{\ell}^{2}}\right|_{0} \Delta a_{\ell}+\left.\frac{\partial \mathcal{C}}{\partial a_{\ell}}\right|_{0} \delta_{\Delta s}+\left.\frac{\partial \mathcal{C}}{\partial a_{\ell}}\right|_{0} \delta_{w}
$$

The notation $\left.\right|_{0}$ signifies $d(t)=0, a_{k}(t)=\frac{s^{k}(t)}{k !}$.

- The term $\left.\frac{\partial^{2} \mathcal{C}}{\partial a_{\ell}^{2}}\right|_{0} \Delta a_{\ell}$, in (3) corresponds to the effect of the coefficient estimation error, $\Delta a_{\ell}$, on the derivative of the cost function.

- In the term $\left.\frac{\partial \mathcal{C}}{\partial a_{\ell}}\right|_{0} \delta_{\Delta s}$, the notation, $\Delta s$ is used to denote the effect of all the derivatives of $s(t)$ greater than $(p+1)$.
- The term, $\left.\frac{\partial \mathcal{C}}{\partial a_{\ell}}\right|_{0} \delta_{w}$ is the contribution of additive noise.

The detailed calculations of the derivatives and the eventual bias and variance expressions are given in Appendix B. The final expressions for the bias and variance are given below.

$$
\begin{aligned}
& \operatorname{Bias}(\widehat{s}(0))=\frac{(-1)^{p+1} s^{p+1}(0)}{(p+1) !} \frac{\int \beta^{p+1} h(\beta) \mathrm{d} \beta}{\int h(\beta) \mathrm{d} \beta} \\
& \operatorname{Var}(\widehat{s}(0))=\sigma_{w}^{2} \frac{\int h^{2}(\beta) \mathrm{d} \beta}{\left[\int h(\beta) \mathrm{d} \beta\right]^{2}}
\end{aligned}
$$

Similar asymptotic expressions for bias and variance were encountered in the case of uniformly sampled time series. The main link here is that, asymptotically, that is, as the sampling density increases, the differences between uniform and nonuniform sampling tend to disappear and the analysis reflects the properties of the underlying continuous-time signal.

\subsection{Optimum window length}

The mean-square error of the estimate is

$$
\begin{aligned}
\operatorname{MSE}(\widehat{s}(0)) & =\mathcal{E}\left\{[s(0)-\widehat{s}(0)]^{2}\right\}=[\operatorname{Bias}(\widehat{s}(0))]^{2}+\operatorname{Var}(\widehat{s}(0)), \\
& =\left(\frac{s^{p+1}(0)}{2^{p+1}(p+2) !}\right)^{2} \sigma_{h}^{2 p+2}+\frac{\sigma_{w}^{2}}{\sigma_{h}}=\mathcal{B} \sigma_{h}^{2 p+2}+\frac{\sigma_{w}^{2}}{\sigma_{h}} .
\end{aligned}
$$

The optimum window parameter, $\sigma_{h_{\mathrm{opt}}}$ is obtained as the minimizer of the MSE:

$$
\sigma_{h_{\mathrm{opt}}}=\arg \min _{\sigma_{h}} \operatorname{MSE}(\widehat{s}(0))=\left[\frac{\sigma_{w}^{2} 2^{2 p+2}((p+2) !)^{2}}{(2 p+2)\left(s^{p+1}(0)\right)^{2}}\right]^{\frac{1}{2 p+3}} .
$$

The optimum window parameter requires the higher-order derivative of the signal, which is not available a priori. Therefore, we develop a nearly optimal approach to compute the window width.

\section{Optimizing window length using the ICI technique}

In the ICI technique, the confidence intervals of the estimator corresponding to each window length are computed using the variance expression. By comparing confidence intervals corresponding to different window lengths and examining them for consistency, the optimum window length is determined. As the analyses in section 2.2 and section 3.1 show, asymptotically, the estimator $\widehat{s}_{L}$ is a 
Gaussian random variable distributed around $s$ with bias $\mathcal{E}\left\{\Delta s_{L}\right\}$ and standard deviation $\sigma_{L}$. Thus, for a fixed noise variance $\sigma_{w}^{2}$, one can write: $\left|s-\widehat{s}_{L}\right| \leq\left|\mathcal{E}\left\{\Delta s_{L}\right\}\right|+\kappa \sigma_{L}$. For $L<L_{\text {opt }}$, if $\left|\mathcal{E}\left\{\Delta s_{L}\right\}\right| \leq \Delta \kappa \sigma_{L}$, the inequality may be rewritten as $\left|s-\widehat{s}_{L}\right| \leq(\Delta \kappa+\kappa) \sigma_{L}$. Therefore, the confidence interval for the signal estimate computed using window length $L_{s}$ is $D_{s}=\left[\widehat{s}_{L_{s}}-(\Delta \kappa+\kappa) \sigma_{L_{s}}\right.$, $\left.\widehat{s}_{L_{s}}+(\Delta \kappa+\kappa) \sigma_{L_{s}}\right]$.

\subsection{Outline of the ICI technique}

Define a set of discrete-window lengths in arithmetic progression, $\mathcal{H}=\left\{L_{s+1}=L_{s}+2, s=0,1,2, \ldots, J ; L_{0}=p+1\right\}$.

1. Initialization: Set $\ell=1$. The estimate $\widehat{s}_{L_{\ell}}$ is obtained using the window length $L_{\ell}$, that is, the noisy data (after data-centering) within the window is used to perform a $p$ th-order fit to estimate the signal at the center of the window.

2. Confidence interval computation: The end-points of the confidence interval are

$$
P_{\ell}=\widehat{s}_{L_{\ell}}-(\kappa+\Delta \kappa) \sigma_{L_{\ell}}, \text { and } Q_{\ell}=\widehat{s}_{L_{\ell}}+(\kappa+\Delta \kappa) \sigma_{L_{\ell}} .
$$

3. Estimation: Obtain $\widehat{s}_{L_{\ell+1}}$ using $L_{\ell+1}=2_{\ell+2}$ in the set $\mathcal{H}$. The end-points of the confidence interval are computed as

$$
\begin{aligned}
P_{\ell+1} & =\widehat{s}_{L_{\ell+1}}-(\kappa+\Delta \kappa) \sigma_{L_{\ell+1}} \text { and } \\
Q_{\ell+1} & =\widehat{s}_{L_{\ell+1}}+(\kappa+\Delta \kappa) \sigma_{L_{\ell+1}} .
\end{aligned}
$$

4. Check: Is $\left[P_{s}, Q_{s}\right] \cap\left[P_{s+1}, Q_{s+1}\right] \neq \emptyset$ ? $(\emptyset$ denotes the empty set). In other words, the following condition is checked for

$$
\left|\widehat{s}_{L_{\ell+1}}-\widehat{s}_{L_{\ell}}\right| \leq 2(\kappa+\Delta \kappa)\left[\sigma_{L_{\ell}}+\sigma_{L_{\ell+1}}\right] .
$$

The smallest value of $\ell$ for which the condition is not satisfied yields the optimum window length that is, if $\ell^{*}$ is the smallest value of $\ell$ for which the condition is not satisfied, then $L_{\mathrm{opt}}=L_{\ell^{*}+1}$, else $\ell \leftarrow \ell+1$, and steps 3 and 4 are repeated.

For $p^{\text {th }}$-order polynomial fit, $L_{0}$ is chosen as $(p+1)$ to ensure that, at any stage in the algorithm, there is sufficient data for performing $p^{\text {th }}$-order polynomial regression.

\subsection{Optimal choice of $\kappa+\Delta \kappa$}

The success of the ICI algorithm depends on the choice of the confidence-interval parameter $\kappa+\Delta \kappa$. Although the parameter may be fixed empirically, we propose a systematic formulation to compute the optimal value by considering a new criterion that takes into account the probability of false alarm to determine the optimal parameter, based on the following observations:
1. The ICI technique is based on the property of cessation of overlap between the confidence intervals corresponding to successive window widths. While the cessation of overlap between the confidence intervals is a necessary condition, it is not sufficient for arriving at a window length close to the true optimum. This can be explained by considering the algorithm performance for a large value of $\kappa+\Delta \kappa$. In this case, the transition from overlap to no overlap will still happen, but corresponding to a window length far away from the optimum, and hence larger MSE. Stankovic [22] also remarks that one should avoid choosing large values of $\kappa+\Delta \kappa$. This is true, but one would never know an appropriate value of the parameter. For small choice of the parameter, the transition will again happen but corresponding to a window length much smaller than the optimum.

2. We also require the probability of a false result to be low. Assuming the error to be Gaussian distributed, the probability of false alarm $\left(P_{F A}\right)$ is [22] $P_{F A}=$ $\operatorname{erfc}\left(\frac{\kappa+\Delta \kappa}{\sqrt{2}} \frac{1+\alpha}{\sqrt{1+\alpha^{2}}}\right), \quad$ where $\quad \alpha=\frac{\sigma\left(h_{s-1}\right)}{\sigma\left(h_{s}\right)}=2^{\frac{m}{2}}, \quad$ and erfc $(x)=\frac{2}{\sqrt{\pi}} \int_{x}^{\infty} e^{-t^{2}} \mathrm{~d} t$. The probability of false alarm is a monotonically decreasing function of $\kappa+\Delta \kappa$, which implies that an arbitrarily low probability of false alarm can be achieved by choosing a large value of $\kappa+\Delta \kappa$. However, as mentioned above, this will yield a window length much larger than the optimum window length and the resulting MSE will be high due to large bias.

Keeping the above aspects in view, we see that the minimum window transition from overlap to no overlap and the probability of false alarm below a certain threshold are important necessary conditions for the algorithm to offer good performance, but they are not sufficient. Therefore, we incorporate the additional constraint that, the least value of $(\kappa+\Delta \kappa)$ that satisfies both the conditions is optimum. This is specified by the coverage area within the $(\kappa+\Delta \kappa)$ wide confidence interval. For Gaussian distributed error, the area is erf $\left(\frac{\kappa+\Delta \kappa}{\sqrt{2}}\right)$. Taking the two conditions together, the new criterion for optimum choice of $\kappa+\Delta \kappa$ is specified as

$$
(\kappa+\Delta \kappa)_{\mathrm{opt}}=\arg \min _{\kappa+\Delta \kappa}\left\{P_{F A}+\operatorname{erf}\left(\frac{\kappa+\Delta \kappa}{\sqrt{2}}\right)\right\} .
$$

\subsection{Experimental corroboration}

The signal definition used is the same as that in [22]. The signal is given by $s(t)=\frac{1}{1+\left(\frac{t}{7.5}\right)^{40}}$ within the interval $|t| \leq 25$ with a sampling interval $\Delta t=\frac{1}{25}$. The signal is 
corrupted by a zero-mean stationary Gaussian additive noise of variance $\sigma_{w}^{2}$. We have used $\sigma_{w}=0.1$. The median noise variance estimator [22] is used for confidence interval computation. For this signal definition, $m=1$. The two components of the new cost function (see (4)), namely, the probability of false alarm and the area function are shown in figure 5 together with their sum. The inset plot is a zoomed-in portion of the cost function about the minimum. The choice of $\kappa+\Delta \kappa$ introduces a trade-off between the area function and probability of false alarm. However, unlike the bias-variance trade-off, this is manageable since the two components being traded for are known deterministically. A closed-form solution may not be possible, but the cost function can be computed on a fine grid to obtain the optimum $(\kappa+\Delta \kappa)$. The optimum value was found to be 1.47 for the example considered.

To verify and corroborate the above value, Monte Carlo simulations are performed with the denoising experiment repeated 100 times for different choices of $(\kappa+\Delta \kappa)$. The average signal-to-estimation-noise ratio is plotted in decibels (dB) along with the spread about the average value, in figure 6. The experimentally obtained optimum is close to the true optimum. The performance deteriorates for large values of $(\kappa+\Delta \kappa)$ for the same input SNR. We obtain about 4-5 dB improvement in performance with optimum choice of $\kappa+\Delta \kappa$ against the sub-optimum choices (see figure 6). This is significant improvement in estimation accuracy.

\section{Experimental Results}

\subsection{Damped sinusoids}

Consider the signal $s(t)=e^{-0.005 t} \sin (0.05 t) u(t)$, in additive white Gaussian noise of variance $\sigma_{w}^{2}$. A sampling

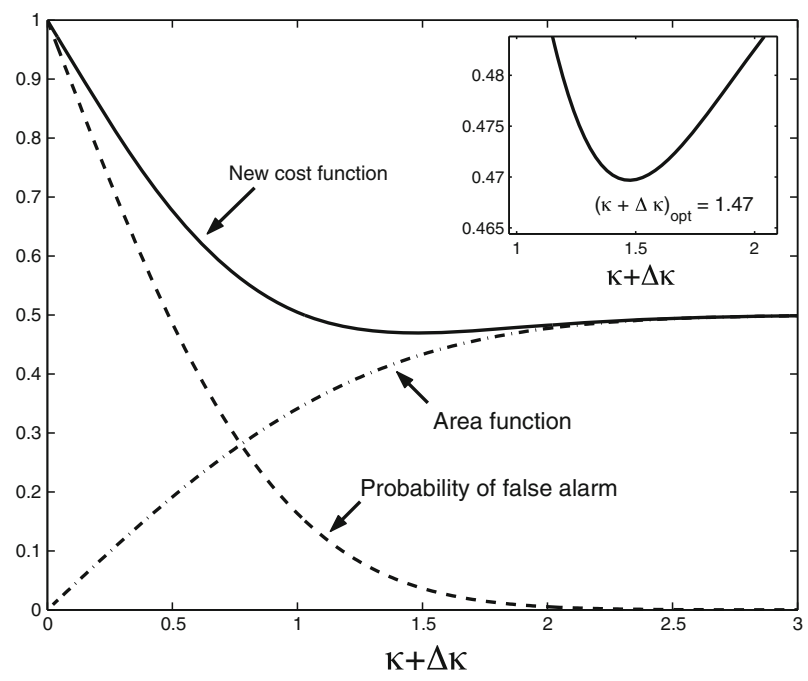

Figure 5. The new cost function for determining optimum $\kappa+\Delta \kappa$.

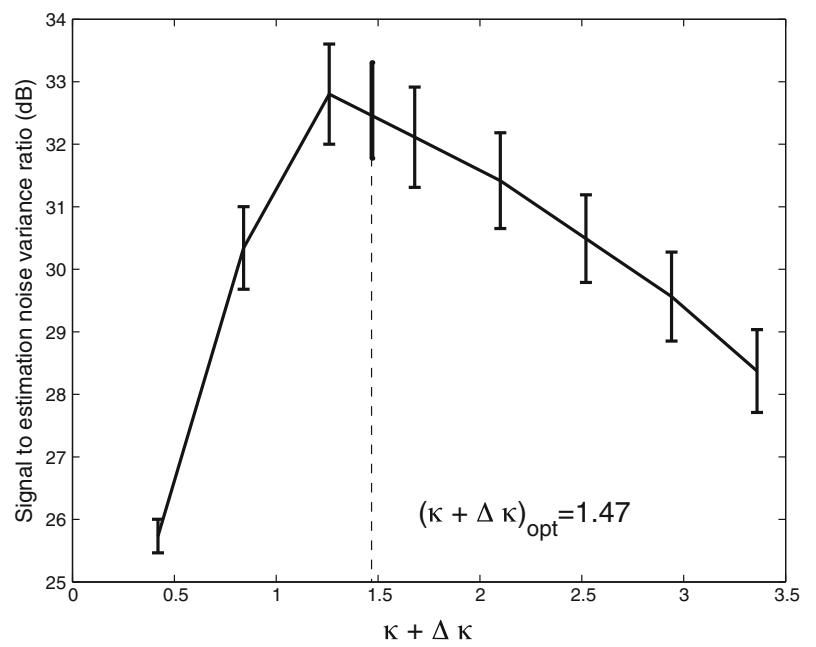

Figure 6. The output signal-to-estimation-noise ratio as a function of $\kappa+\Delta \kappa$, corresponding to an input SNR of $20 \mathrm{~dB}$.

sequence is generated using a uniformly distributed random number generator and is fixed for all the Monte Carlo realizations of the noisy signal. The noisy signal is sampled at 256 nonuniform locations as determined by the sampling sequence. The signal $s(t)$ is estimated at 256 uniform locations $\{0,1,2, \ldots, 255\}$ using the proposed technique. We use a third-order polynomial that is, $p=3$, for STPR. The initial window length is chosen as the interval encompassing $(p+1)$ samples. At each iteration of the technique, the increment is chosen as the window length covering $(p+1)$ new samples. The value of $\kappa+\Delta \kappa$ is set to 1.5 . The experiment is repeated 100 times with different random realizations of the noise sequence. Since the ground truth $s(t)$ is known in the simulation, we compute a measure of accuracy of the estimator by defining a cumulative MSE (CMSE):

$\eta_{\mathrm{opt}}=10 \log _{10}\left(\frac{1}{R(N-20)} \sum_{r=1}^{R} \sum_{n=11}^{N-10}\left[\widehat{s}^{(r)}(n)-s(n)\right]^{2}\right) \mathrm{dB}$,

where $R$ is the total number of realizations, $s^{(r)}(n)$ is the estimate of $s(n)$ in the $r^{\text {th }}$ realization, and $N$ is the number of points at which the estimates are obtained. The estimators at the boundary of the window are excluded in the computation of the CMSE because they are large due to truncation effects. The measure $\eta_{\text {opt }}$ takes into account the bias and variance at each point in the observation interval. For comparison, we perform STPR based on the $k$-nearest neighbor approach $\eta_{k-N N}$ (with $k=7$ in the experiment). The CMSE corresponding to the $k-N N$ technique is denoted by $\eta_{k-N N}$.

The estimates of the sequence $s(n), 0 \leq n \leq 255$ from the 100 different realizations, using the two techniques, are shown in figure 7 for two different values of $\sigma_{w}$. The proposed technique that yields low-variance estimates offers 

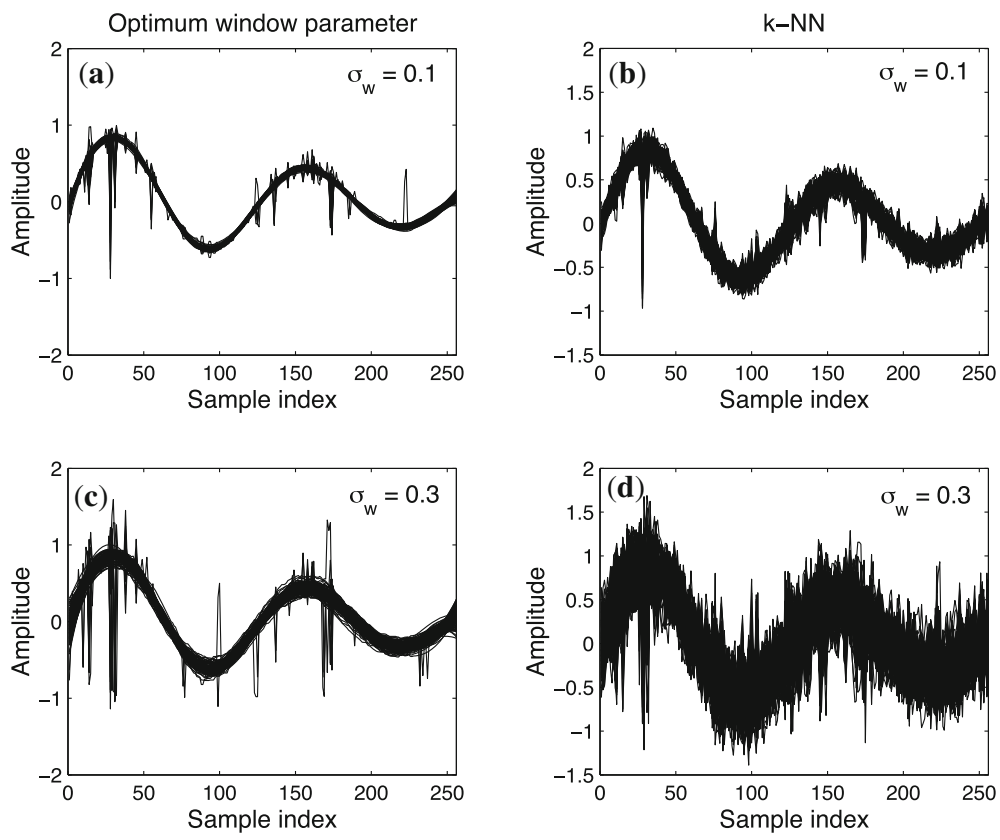

Figure 7. Overlaid estimators from different realizations. The plots (a) and (c) correspond to the optimum window parameter selection for $\sigma_{w}=0.1$ and $\sigma_{w}=0.3$, respectively. The plots $(\mathbf{b})$ and (d) correspond to the $k$-NN technique for $\sigma_{w}=0.1$ and $\sigma_{w}=0.3$, respectively.

higher consistency. The CMSE performance is shown in table 1 and in figure 8 for different values of $\sigma_{w}$. Observe that $\eta_{\text {opt }}$ is about 7-9 dB lesser than $\eta_{k-N N}$. The proposed technique is more robust to noise because it adapts the confidence interval length to the noise variance and yields estimates with near-optimal MSE.

\subsection{Chirp signals}

We next consider a linearly frequency-modulated chirp with an exponentially decaying envelope (by a factor of $\frac{1}{e}$ within the observation window), which may be viewed as a synthesized formant of a speech signal. The frequency is swept from 0.05 to 0.1 (these are normalized frequencies with 0.5 corresponding to the Nyquist frequency). The estimated signal and the MSE performance at the $111^{\text {th }}$ position (an arbitrarily chosen point corresponding to high local SNR) and $200^{\text {th }}$ position (corresponding to low local SNR) in the window are shown in figure 9. The new technique offers accurate estimation for moderate to high SNRs even for time-varying signals. The signal estimation accuracy is higher in regions of high local SNR than in regions of poor local SNR.

\subsection{Sinusoids}

We report experimental results for MMSE signal estimation and discuss the effect of the order of the polynomial. We consider the asymptotic bias and variance expressions for a
Table 1. CMSE comparison of the adaptive window and $k-N N$ techniques.

\begin{tabular}{llc}
\hline$\sigma_{w}$ & $\eta_{\text {opt }} \mathrm{dB}$ & $\eta_{k-N N} \mathrm{~dB}$ \\
\hline 0.10 & -26.24 & -20.26 \\
0.15 & -24.41 & -17.62 \\
0.20 & -23.80 & -15.69 \\
0.25 & -22.39 & -13.77 \\
0.30 & -21.55 & -12.42 \\
\hline
\end{tabular}

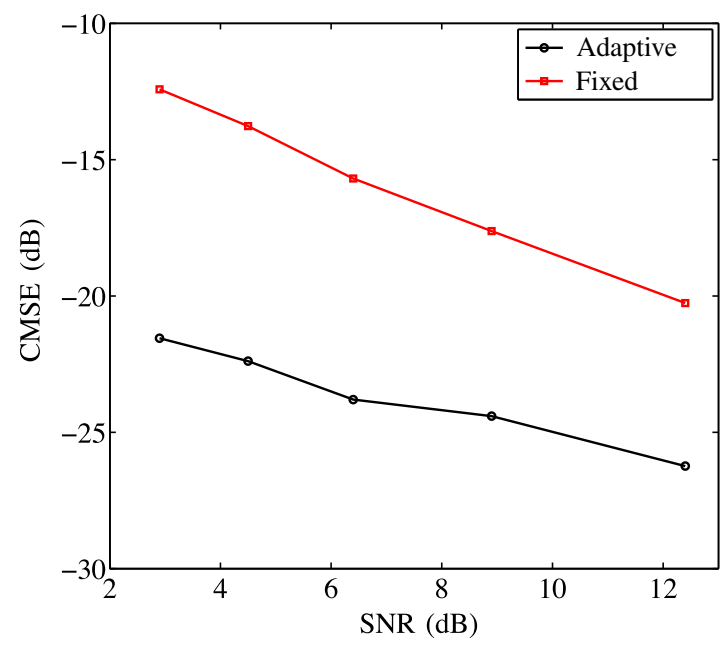

Figure 8. CMSE for fixed vs. adaptive window techniques as a function of the SNR. 

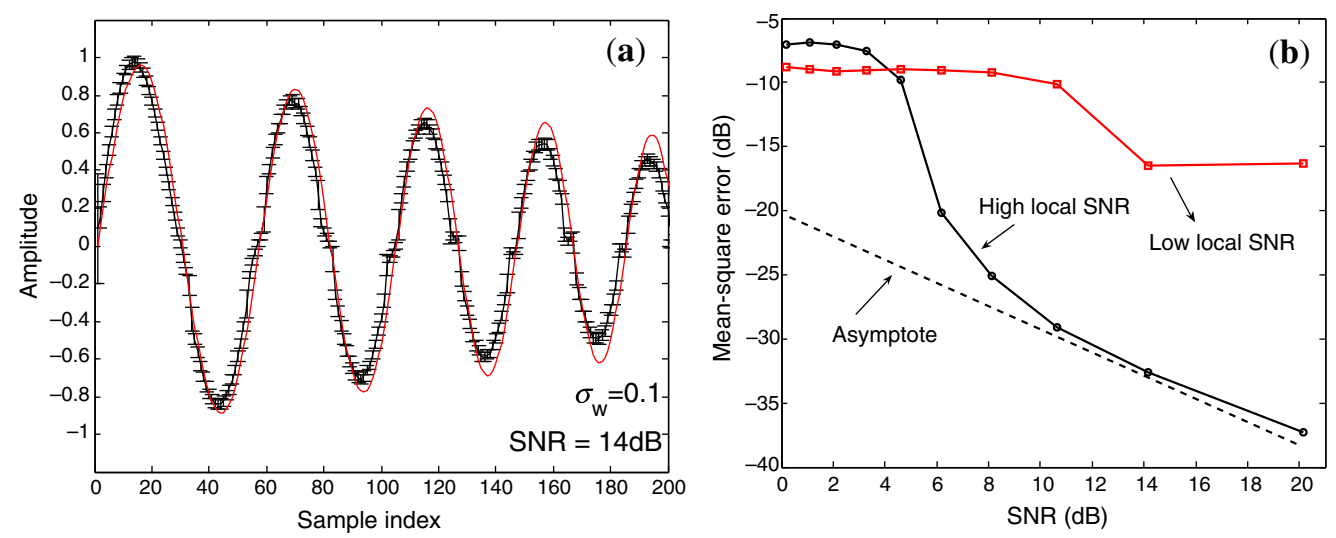

Figure 9. (a) The estimated signal with the $2 \sigma$-confidence interval and the actual signal and (b) MSE as a function of the SNR.

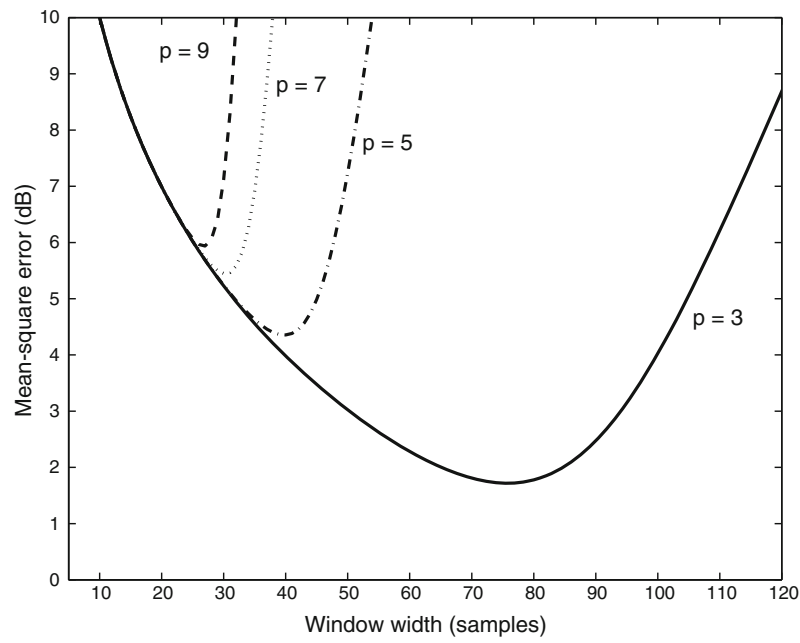

Figure 10. MSE versus window length as a function of the polynomial order.

sinusoid $s[n]=10^{-5} \sin \left(2 \pi f_{0} n\right), 0 \leq n \leq 1000, f_{0}=0.2$, in noise of standard deviation, $\sigma_{w}=10$. At an arbitrary chosen location, $n=259$, we plot the squared bias and variance (cf. figure 10) as a function of the window length for $p=3,5,7$, and 9 .

The MSE varies slowly as a function of the window length, for lower-order polynomials. For higher-order polynomials, the MSE varies rapidly about the optimum window length. Also, as the polynomial order increases, the optimum window length shifts to the left with a corresponding increase in the MSE. For high orders, the optimum window length is small; this might lead to a numerically ill-posed set of equations. The curve corresponding to $p=1$ is not shown because the optimum was found to occur for a much larger value of the window length requiring large data sizes. Also, a straight-line model corresponding to $p=1$ may not be suitable for real-world
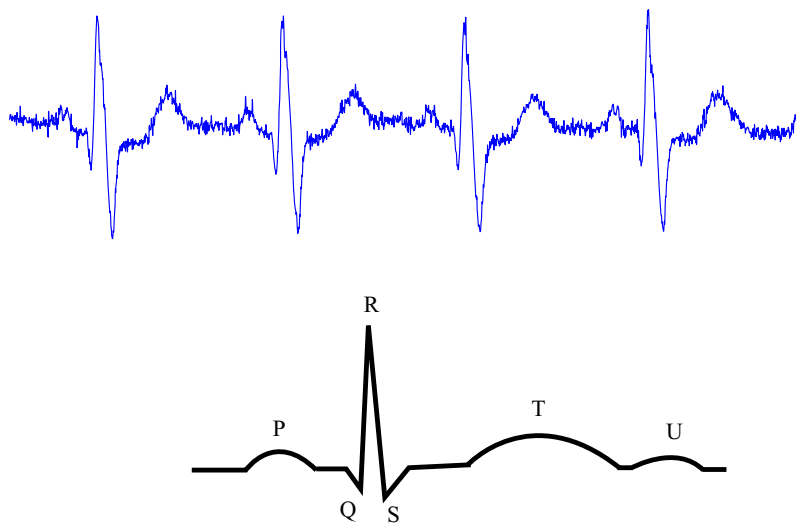

Figure 11. A noisy ECG signal and a stylized representation of one cycle to justify short-time polynomial representation.

signals over large window lengths. In our experiments, we chose $p=3$. While the exact nature of the curves depends on the signal and the noise variance, the observations apply in general to smooth signals. The important aspect here is that, in searching for the optimum window length, a coarse search using the dyadic set of windows might yield a higher MSE than that obtained with window lengths in arithmetic progression that is, $\mathcal{H}=\left\{L_{s+1}=L_{s}+2, s=0,1,2, \ldots, J\right\}$. In the literature, a dyadic set of windows has been used $[8,9,23]$, but by choosing a set of arithmetic windows, the search space for the window lengths is finer and hence the accuracy will be higher. $L_{0}=p+1$ for initialization and $J$ is determined such that $L_{J+1}$ just equals or exceeds the data size. A window length of $L_{s}$ implies $0.5 L_{s}$ samples on either side of the instant at which the estimate is desired. We used a $5 \sigma$-confidence interval $(\kappa+\Delta \kappa=2.5)$, with the corresponding coverage probability equal to 0.99 . This value of $\kappa+\Delta \kappa$ also yielded good results in other estimation problems [8, 23, 24]. 

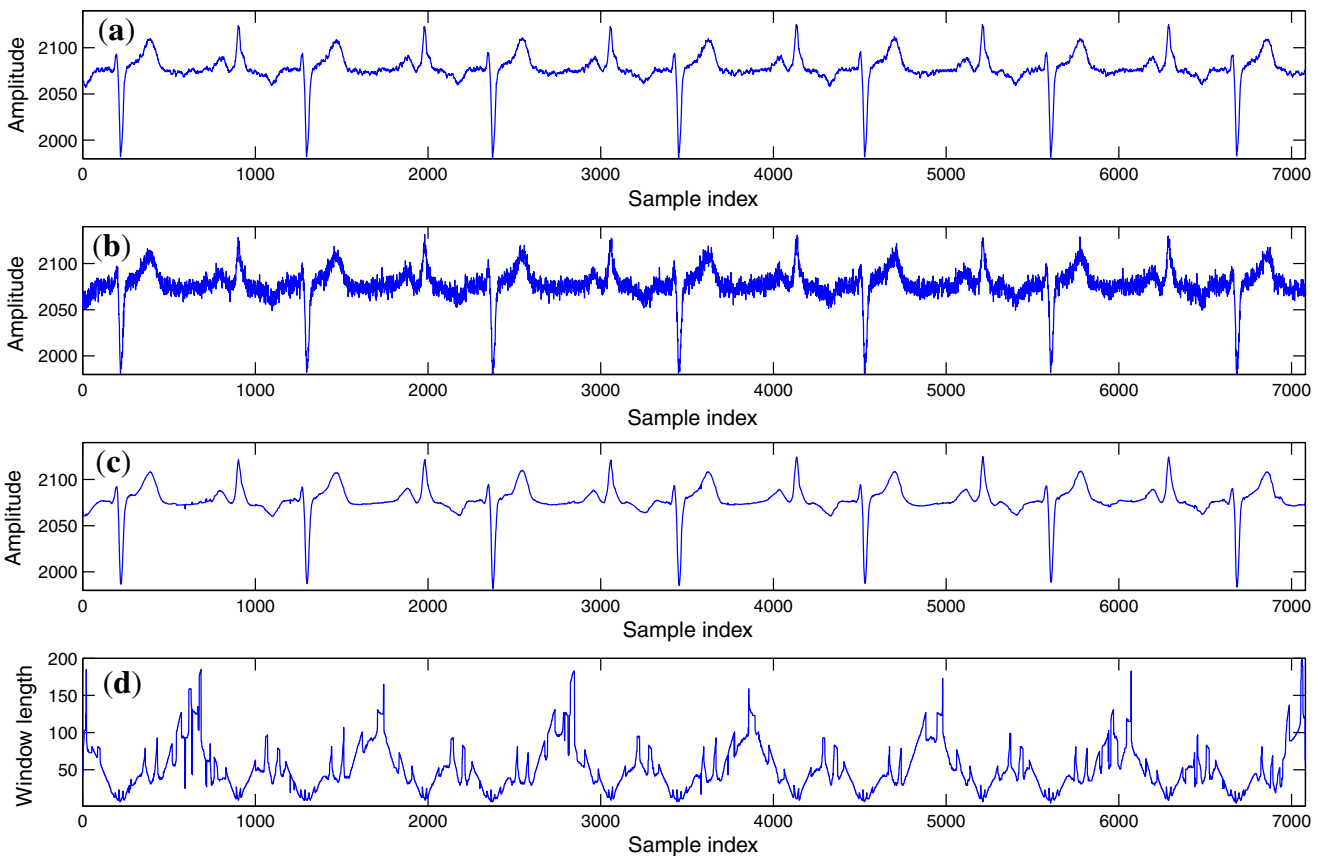

Figure 12. (a) An ECG recording, (b) noisy ECG recording, (c) estimated ECG signal, and (d) instantaneous window lengths.
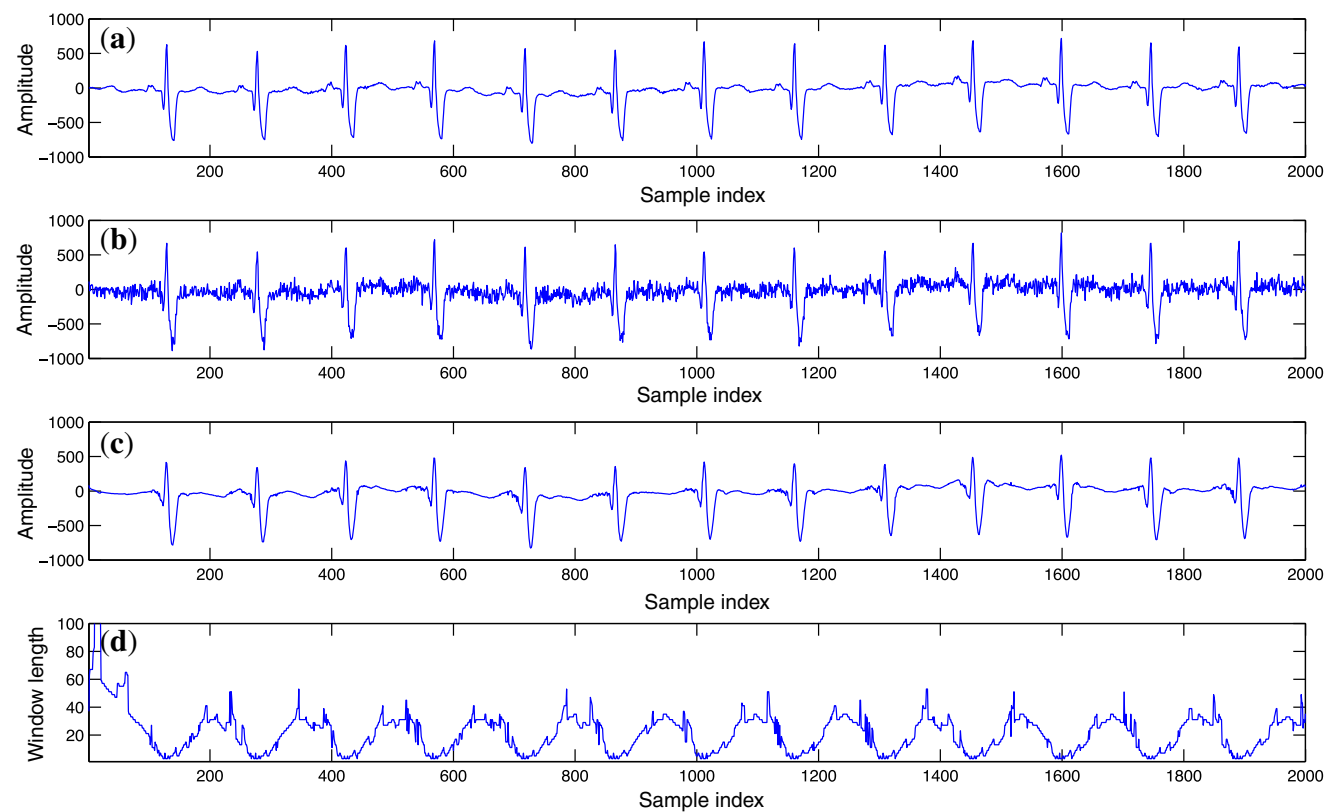

Figure 13. (a) An ECG recording, (b) noisy ECG recording, (c) estimated ECG signal, and (d) instantaneous window lengths.

\subsection{Electrocardiogram (ECG) signals}

ECG signals are typically quasi-periodic and possess a characteristic temporal structure. High-quality ECG recordings are preferred for their diagnostic value and clinical significance. The main sources of noise in ECG signals are high-frequency noise caused by electromyogram-induced noise, power-line interferences (line noise), 

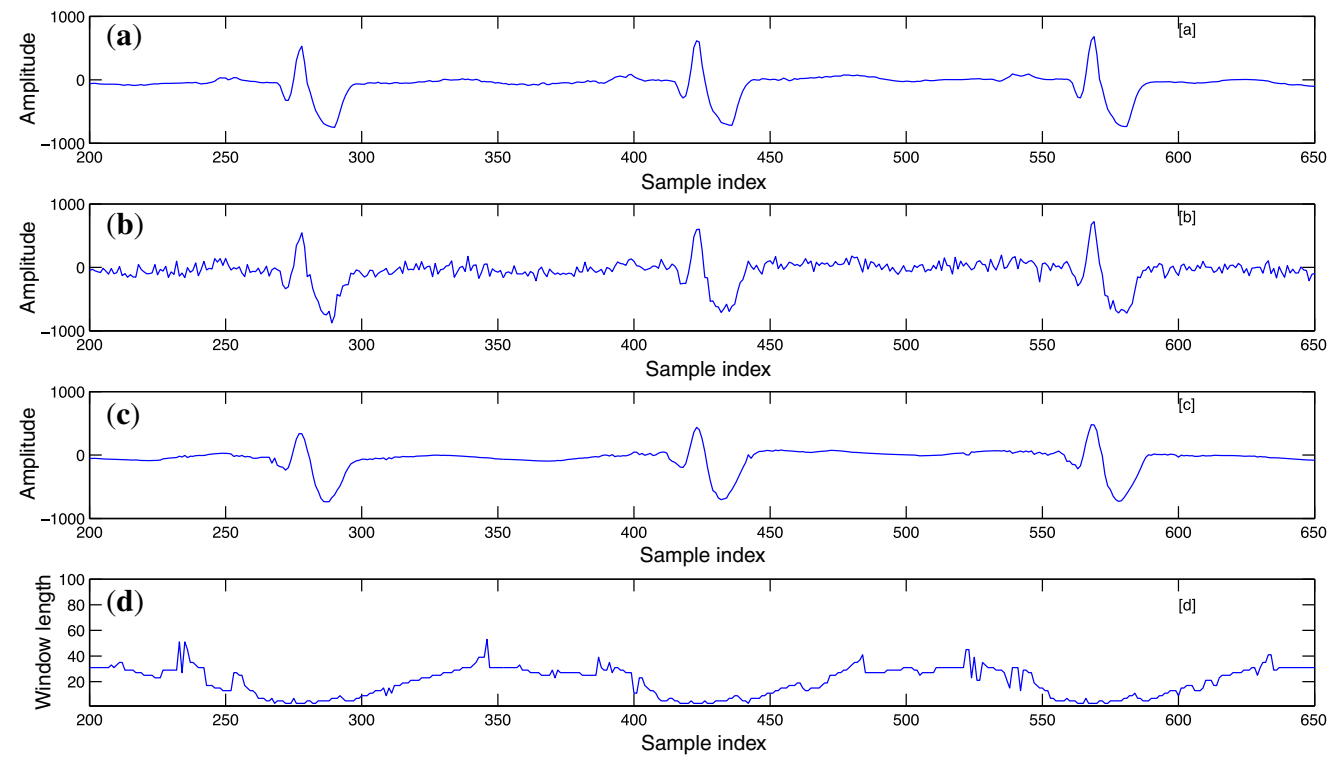

Figure 14. Zoomed-in portions of the signals in Figure 13; (a) an ECG recording, (b) noisy ECG recording, (c) estimated ECG signal, and (d) instantaneous window lengths.

mechanical forces acting on the electrodes (electrode artifacts), artifacts due to respiration, etc. Our goal in this experiment is to exploit the smooth temporal structure and apply the short-time polynomial model for representing ECG signals. The suitability of the STPR for ECG signals is justified by comparing the real ECG signal with a stylized piecewise-polynomial representation shown in figure 11. In fact, the piecewise-polynomial aspect of the ECG waveform is often used to synthesize test signals ${ }^{1}$ with user-settable parameters such as heart rate, signal duration, sampling frequency, QRS amplitude and duration, and T-wave amplitude.

The ECG samples are taken from the PhysioBank database (sampling frequency of $720 \mathrm{~Hz})^{2}$. An example is shown in figure 12(a) and its noisy version (additive white Gaussian noise, SNR = $10.46 \mathrm{~dB}$ ) is shown in figure 12(b). The ECG signal estimated by STPR is shown in figure 12(c). The instantaneous window lengths are shown in figure 12(d). The figures show a significant improvement in the SNR. Comparing the reconstruction with the ground truth, one finds that the temporal structure of the waveform is also preserved. From figure 12(d) suggests that the instantaneous window lengths are optimally chosen by the proposed approach. In regions of fast signal transitions, the ICI technique picks short windows, and in steady-state regions, it selects longer windows.

Another set of results on a pathological ECG signal ${ }^{3}$ is shown in figure 13 and a zoomed-in plot of the ECG signal is shown in figure 14. The small signal transitions and

\footnotetext{
${ }_{1}^{1}$ http://www.physionet.org/physiotools/matlab/ECGwaveGen/

2 http://www.physionet.org/physiobank/database/aami-ec13/.

${ }^{3}$ http://www.physionet.org/physiobank/database/ptbdb/patient001/ s0010_re.hea
}

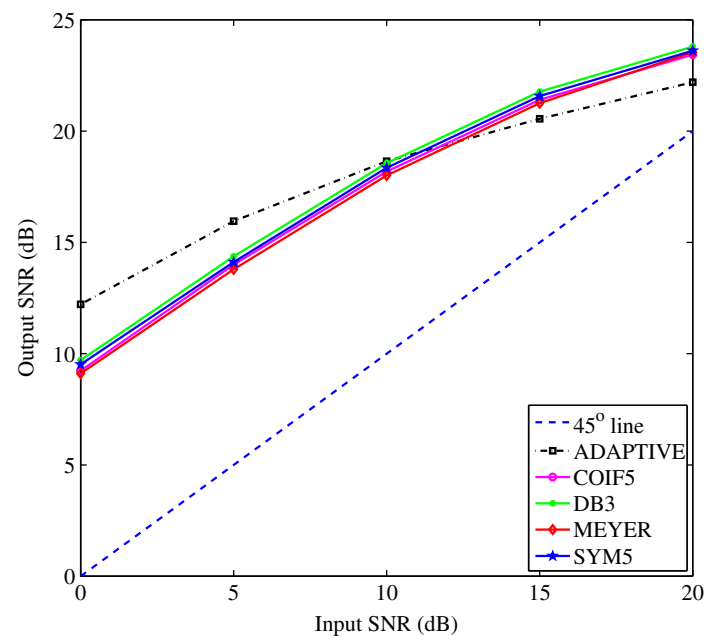

Figure 15. Performance comparison of the adaptive STPR technique with wavelet denoising techniques; (COIF: Coiflet, DB: Daubechies, MEYER: Meyer wavelet, SYM: Symlets).

shape are retained by the algorithm and the noise is suppressed to a large extent.

\subsection{Comparison with wavelet denoising techniques}

We next compare the denoising performance with standard wavelet techniques employing soft-thresholding [25] (cf. figures 15, 16, and 17). A standard three-level decomposition was performed in all the cases. The adaptive regression technique is consistently superior by about 2 to 3 $\mathrm{dB}$ for low SNR. For high SNR, the wavelet techniques are more accurate. These results indicate that the adaptive STPR technique has better performance at low SNRs. 


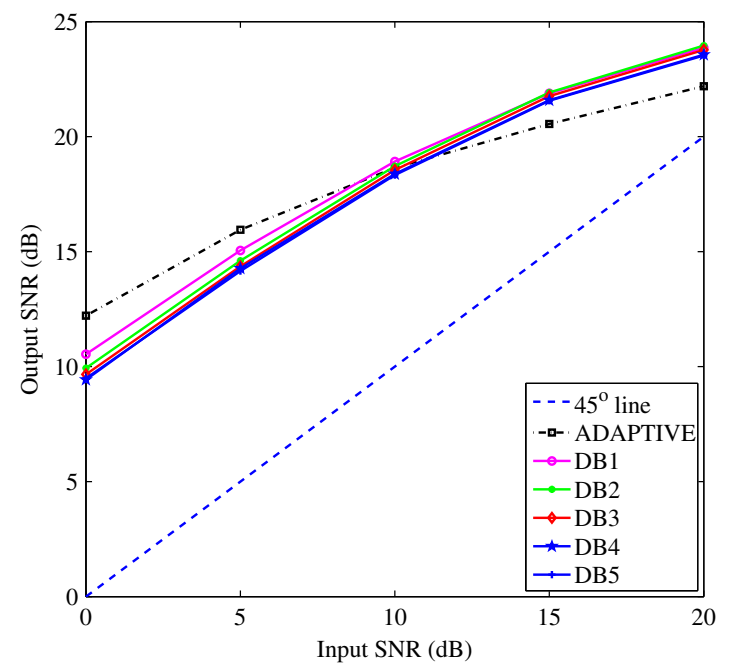

Figure 16. Performance comparison of the adaptive STPR technique with wavelet denoising techniques; Wavelets: DB1 to DB5 (DB: Daubechies).

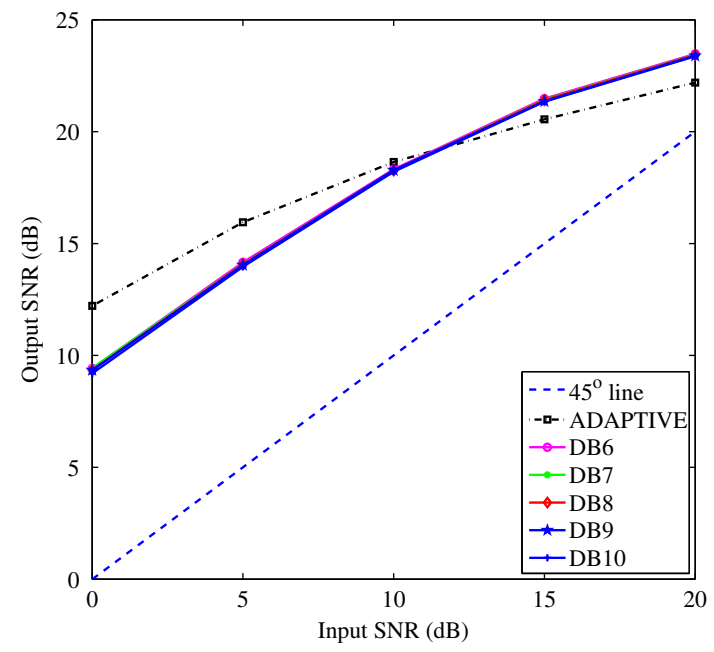

Figure 17. Performance comparison of the adaptive STPR technique with wavelet denoising techniques; Wavelets: DB6 to DB10 (DB: Daubechies).

\section{Conclusions}

We have addressed the problem of optimum short-time polynomial representation of signals, and derived the biasvariance trade-off as a function of the window length and obtained expressions for the optimal window length. The trade-off is solved within the framework of a stochastic algorithm based on the intersection-of-confidence-intervals technique. Our problem formulation is in the time domain and does not assume the signal to be stationary or quasistationary. Experimental results show that this feature makes the technique effective for modeling time-varying signals. The performance of the technique is assessed for adaptive regression and denoising of ECG signals in the presence of noise. Experimental results showed a significant SNR improvement. At $10 \mathrm{~dB}$ input SNR, the gain achieved is about $8 \mathrm{~dB}$. We have also carried out a performance comparison with basic wavelet denoising techniques. The advantage of the proposed technique over the wavelet approaches is mainly in the low SNR regime (SNR less than $10 \mathrm{~dB}$ ) where the SNR gain with the proposed technique is about $3 \mathrm{~dB}$ higher. At SNR greater than $10 \mathrm{~dB}$, the wavelet techniques outperform the proposed technique by about $3 \mathrm{~dB}$.

The methodology proposed in this paper could also be used for related problems such as kernel density estimation, which is often encountered in pattern recognition and machine learning; scattered data interpolation, which one comes across in many engineering disciplines; denoising of images by considering a suitable two-dimensional extension of the short-time polynomial modeling approach.

\section{Acknowledgements}

This work was supported in part by the Department of Science and Technology, India project for Intensification of Research in High-Priority Areas (DST-IRHPA) grant.

\section{Appendix}

\section{A. Uniform sampling: bias and variance calculations}

Based on the cost function given in section (2.2), the partial derivatives are evaluated next:

$$
\begin{aligned}
& \left.\frac{\partial \mathcal{C}}{\partial a_{\ell}}\right|_{0}=0 \quad \text { (optimum corresponding to zero estimation error), } \\
& \left.\frac{\partial^{2} \mathcal{C}}{\partial a_{\ell}^{2}}\right|_{0} \Delta a_{\ell}=2 \sum_{n=m T-L / 2}^{m T+L / 2}(m T-n T)^{2 \ell} h(n T) \Delta a_{\ell}, \\
& \left.\frac{\partial \mathcal{C}}{\partial a_{\ell}}\right|_{0} \delta_{\Delta s}=-2 \sum_{n=m T-L / 2}^{m T+L / 2} \Delta s(m T, n T)(m T-n T)^{\ell} h(n T), \text { and } \\
& \left.\frac{\partial \mathcal{C}}{\partial a_{\ell}}\right|_{0} \delta_{w}=-2 \sum_{n=m T-L / 2}^{m T+L / 2} w(m T-n T)(m T-n T)^{\ell} h(n T) .
\end{aligned}
$$

Since the signal model takes care of polynomials up to order $p$, the model mismatch error is expressed by using a Taylor series approximation for $\Delta s(m T, n T)$. Truncating the approximation beyond order $p+2$, we get that

$$
\Delta s(m T, n T)=\frac{(-n T)^{(p+1)}}{(p+1) !} s^{(p+1)}(m T)+\frac{(-n T)^{(p+2)}}{(p+2) !} s^{(p+2)}(m T),
$$

where $p$ ! denotes the factorial of $p$ and $s^{(k)}(m T)$ denotes the $k^{\text {th }}$ derivative of $s(t)$, evaluated at $t=m T$. To obtain 
minimum fitting error, we set $\frac{\partial \mathcal{C}}{\partial a_{\ell}}, 0 \leq \ell \leq p$, to zero. Using

(1) gives ${ }^{4}$

$$
\begin{aligned}
\Delta a_{\ell}= & \frac{\frac{(-1)^{p+1} s^{(p+1)}(m T)}{(p+1) !} \sum_{n}(n T)^{p+1}(m T-n T)^{\ell}}{\sum_{n}(m T-n T)^{2 \ell}} \\
& +\frac{\frac{(-1)^{p+2} s^{(p+2)}(m T)}{(p+2) !} \sum_{n}(n T)^{p+2}(m T-n T)^{\ell}}{\sum_{n}(m T-n T)^{2 \ell}} \\
& +\frac{\sum_{n} w(t-n T)(m T-n T)^{\ell}}{\sum_{n}(m T-n T)^{2 \ell}} .
\end{aligned}
$$

The bias of the coefficient estimator is $a_{\ell}-\mathcal{E} \widehat{a}_{\ell}=\mathcal{E}\left\{\Delta a_{\ell}\right\}$ and is obtained by taking expectation on both sides of (5):

$$
\begin{aligned}
& \mathcal{E}\left\{\Delta a_{\ell}\right\}=\frac{\frac{(-1)^{p+1} s^{(p+1)}(m T)}{(p+1) !} \sum_{n}(n T)^{p+1}(m T-n T)^{\ell}}{\sum_{n}(m T-n T)^{2 \ell}} \\
& +\frac{\frac{(-1)^{p+2} s^{(p+2)}(m T)}{(p+2) !} \sum_{n}(n T)^{p+2}(m T-n T)^{\ell}}{\sum_{n}(m T-n T)^{2 \ell}}, 0 \leq \ell \leq p
\end{aligned}
$$

The covariance of the coefficient errors is $\operatorname{Cov}\left(\Delta a_{\ell}, \Delta a_{q}\right)=\mathcal{E}\left\{\left(\Delta a_{\ell}-\mathcal{E} \Delta a_{\ell}\right)\left(\Delta a_{k}-\mathcal{E} \Delta a_{k}\right)\right\}$. Representing $\mathcal{E}\{w(m T-n T) w(m T-k T)\}=r_{w w}((n-k) T)$, we write

$$
\operatorname{Cov}\left(\Delta a_{\ell}, \Delta a_{q}\right)=\frac{\sum_{n} \sum_{k}(m T-n T)^{\ell}(m T-k T)^{q} r_{w w}((n-k) T)}{\sum_{n} \sum_{k}(m T-n T)^{2 \ell}(m T-k T)^{2 q}} .
$$

If $r_{w w}((n-k) T)=\sigma_{w}^{2} \delta_{n, k}$, (uncorrelated noise)

$$
\operatorname{Cov}\left(\Delta a_{\ell}, \Delta a_{q}\right)=\sigma_{w}^{2} \frac{\sum_{m}(m T-k T)^{\ell+q}}{\sum_{n} \sum_{k}(m T-n T)^{2 \ell}(m T-k T)^{2 q}} .
$$

$$
\begin{aligned}
& \text { The } \begin{array}{c}
\text { variance } \\
\sum_{k}(m T-k T)^{2 \ell}
\end{array} \\
& \frac{\text { is }}{\sum_{n} \sum_{k}(m T-n T)^{2 \ell}(m T-k T)^{2 \ell}}=\frac{\sigma_{w}^{2}}{\sum_{n}(m T-n T)^{2 \ell}} .
\end{aligned}
$$

\section{Asymptotic considerations}

Considering fine sampling, that is, $T \rightarrow 0, \frac{T}{L} \rightarrow 0$, we approximate the summations by integrals, as follows.

$$
\begin{aligned}
\mathcal{E}\left\{\Delta a_{\ell}\right\} \rightarrow & \frac{\frac{(-1)^{p+1} s^{(p+1)}(m T)}{(p+1) !} \int_{-L / 2}^{L / 2} \tau^{p+1}(m T-\tau)^{\ell} \mathrm{d} \tau}{\int_{-L / 2}^{L / 2}(m T-\tau)^{2 \ell} \mathrm{d} \tau}+ \\
& +\frac{\frac{(-1)^{p+2} s^{(p+2)}(m T)}{(p+2) !} \int_{-L / 2}^{L / 2} \tau^{p+2}(m T-\tau)^{\ell} \mathrm{d} \tau}{\int_{-L / 2}^{L / 2}(m T-\tau)^{2 \ell} \mathrm{d} \tau}, \text { and } \\
\operatorname{Var}\left(\Delta a_{\ell}\right) \rightarrow & \frac{\sigma_{w}^{2}}{\int_{-L / 2}^{L / 2}(m T-\tau)^{2 \ell} \mathrm{d} \tau} .
\end{aligned}
$$

Substituting the expressions for the bias and variance of the coefficient estimates would yield complicated expressions for the bias and variance for the signal estimates. Considerable simplification is achieved by using the idea of "datacentering," that is, the point of interest $t$ is displaced to the origin and STPR is performed after translating the data about $t=m T$ to the origin. This yields simplified expressions for the bias and variance in several parameter estimation problems [26]. Thanks to the data-centering step, Eq. (6) with $\ell=0$ becomes

$$
\begin{aligned}
& \mathcal{E}\{\Delta s(0)\}=\mathcal{E}\left\{\Delta a_{0}\right\}=\frac{(-1)^{p+1} s^{(p+1)}(0)}{L(p+1) !} \int_{-L / 2}^{L / 2} \tau^{p+1} \mathrm{~d} \tau \\
& +\frac{(-1)^{p+2} s^{(p+2)}(0)}{L(p+2) !} \int_{-L / 2}^{L / 2} \tau^{p+2} \mathrm{~d} \tau \\
& = \begin{cases}\frac{s^{(p+1)}(0)}{(p+2) !}\left(\frac{L}{2}\right)^{p+1} & p \text { odd }, \\
\frac{s^{(p+2)}(0)}{(p+3) !}\left(\frac{L}{2}\right)^{p+2} & p \text { even },\end{cases}
\end{aligned}
$$

and $\operatorname{Var}(\Delta s(0))=\operatorname{Var}\left(\Delta a_{0}\right)=\frac{\sigma_{w}^{2}}{L}$

\section{B. Nonuniform sampling: bias and variance calculations}

With a change of variable from $t_{n}$ to $t-t_{n}$, each of the quantities in (3) are evaluated as follows $(0 \leq \ell \leq p)$ :

\footnotetext{
$\overline{4}$ The bias and (co)variance expressions for $\Delta a_{\ell}$ and $\Delta a_{k}$ hold for $0 \leq \ell, k \leq p$; the summation $\sum_{n}$ means $\sum_{n T=m T-\frac{L}{2}}^{m T+\frac{L}{2}}$.
} 


$$
\begin{aligned}
& \left.\frac{\partial \mathcal{C}}{\partial a_{\ell}}\right|_{0}=-2 \sum_{n}\left[s\left(t-t_{n}\right)-\sum_{k=0}^{p} a_{k}\left(t-t_{n}\right)^{k}\right]\left(t-t_{n}\right)^{\ell} h\left(t_{n}\right)=0, \\
& \left.\frac{\partial^{2} \mathcal{C}}{\partial a_{\ell}^{2}}\right|_{0} \Delta a_{\ell}=2 \sum_{n}\left(t-t_{n}\right)^{2 \ell} h\left(t_{n}\right) \Delta a_{\ell}, \\
& \left.\frac{\partial \mathcal{C}}{\partial a_{\ell}}\right|_{0} \delta_{\Delta s}=-2 \sum_{n} \Delta s\left(t, t_{n}\right)\left(t-t_{n}\right)^{\ell} h\left(t_{n}\right), \\
& \left.\frac{\partial \mathcal{C}}{\partial a_{\ell}}\right|_{0} \delta_{w}=-2 \sum_{n} w\left(t-t_{n}\right)\left(t-t_{n}\right)^{\ell} h\left(t_{n}\right) .
\end{aligned}
$$

Solving for $\Delta a_{\ell}, 0 \leq \ell \leq p$ gives that

$$
\begin{aligned}
\Delta a_{\ell}= & \frac{(-1)^{p+1} s^{(p+1)}(t)}{(p+1) !}\left[\frac{\sum_{n} t_{n}^{p+1}\left(t-t_{n}\right)^{\ell} h\left(t_{n}\right)}{\sum_{n}\left(t-t_{n}\right)^{2 \ell} h\left(t_{n}\right)}\right] \\
& +\frac{\sum_{n} d\left(t-t_{n}\right)\left(t-t_{n}\right)^{\ell} h\left(t_{n}\right)}{\sum_{n}\left(t-t_{n}\right)^{2 \ell} h\left(t_{n}\right)} .
\end{aligned}
$$

The bias of the estimate $\widehat{a}_{\ell}$ is

$$
\mathcal{E}\left\{\Delta a_{\ell}\right\}=\frac{(-1)^{p+1} s^{(p+1)}(t)}{(p+1) !}\left[\frac{\sum_{n} t_{n}^{p+1}\left(t-t_{n}\right)^{\ell} h\left(t_{n}\right)}{\sum_{n}\left(t-t_{n}\right)^{2 \ell} h\left(t_{n}\right)}\right] .
$$

The covariance of $\widehat{a}_{\ell}$ and $\widehat{a}_{k}$ is

$$
\operatorname{Cov}\left(\Delta a_{\ell}, \Delta a_{k}\right)=\frac{\sum_{n} \sum_{m} r_{w w}\left(t_{n}, t_{m}\right)\left(t-t_{n}\right)^{\ell}\left(t-t_{m}\right)^{k} h\left(t_{n}\right) h\left(t_{m}\right)}{\sum_{n} \sum_{m}\left(t-t_{n}\right)^{2 \ell}\left(t-t_{m}\right)^{2 k} h\left(t_{n}\right) h\left(t_{m}\right)},
$$

where $r_{w w}\left(t_{n}, t_{m}\right)=\mathcal{E}\left\{w\left(t-t_{n}\right) w\left(t-t_{m}\right)\right\}$. Since noise is white, $r_{w w}\left(t_{n}, t_{m}\right)=\mathcal{E}\left\{w\left(t-t_{n}\right) w\left(t-t_{m}\right)\right\}=\sigma_{w}^{2} \delta\left(t_{n}-t_{m}\right)$. The covariance simplifies to: $\operatorname{Cov}\left(\Delta a_{\ell}, \Delta a_{k}\right)=$ $\sigma_{w}^{2}\left\{\frac{\sum_{n}\left(t-t_{n}\right)^{\ell+k} h^{2}\left(t_{n}\right)}{\sum_{n} \sum_{m}\left(t-t_{n}\right)^{2 \ell}\left(t-t_{m}\right)^{2 k} h\left(t_{n}\right) h\left(t_{m}\right)}\right\}$. The variance of the estimator is $\operatorname{Var}\left(\Delta a_{\ell}\right)=\sigma_{w}^{2}\left\{\frac{\sum_{n}\left(t-t_{n}\right)^{2 \ell} h^{2}\left(t_{n}\right)}{\left[\sum_{n}\left(t-t_{n}\right)^{2} h\left(t_{n}\right)\right]^{2}}\right\}$.

\section{Asymptotic considerations}

Denote the maximum distance between the sampling instants by $\epsilon$ that is, $\epsilon=\max _{n}\left\{t_{n+1}-t_{n}\right\}$. In the limiting case, as $\epsilon \rightarrow 0^{+}$, we have that,

$$
\begin{aligned}
\operatorname{Bias}\left(\Delta a_{\ell}\right) & =(-1)^{p+1} s^{p+1}(t) \frac{\int \beta^{p+1}(t-\beta)^{\ell} h(\beta) \mathrm{d} \beta}{\int(t-\beta)^{2 \ell} h(\beta) \mathrm{d} \beta}, \\
\operatorname{Cov}\left(\Delta a_{\ell}, \Delta a_{k}\right) & =\sigma_{w}^{2} \frac{\int(t-\beta)^{\ell+k} h^{2}(\beta) \mathrm{d} \beta}{\int\left(t-\beta_{1}\right)^{2 \ell}\left(t-\beta_{2}\right)^{2 k} h\left(\beta_{1}\right) h\left(\beta_{2}\right) \mathrm{d} \beta_{1} \mathrm{~d} \beta_{2}}, \\
\operatorname{Var}\left(\Delta a_{\ell}\right) & =\sigma_{w}^{2} \frac{(t-\beta)^{2 \ell} h^{2}(\beta) \mathrm{d} \beta}{\left[\int(t-\beta)^{2 \ell} h(\beta) \mathrm{d} \beta\right]^{2}} .
\end{aligned}
$$

The signal is estimated as $\widehat{s}(t)=\sum_{k=0}^{p} \widehat{a}_{k} t^{k}$. The bias and variance of $\widehat{s}(t)$ are

$$
\begin{aligned}
\operatorname{Bias}(\widehat{s}(t)) & =\sum_{k=0}^{p} \operatorname{Bias}\left(\Delta a_{k}\right) t^{k}, \\
\operatorname{Var}(\widehat{s}(t)) & =\sum_{\ell=0}^{p} \sum_{k=0}^{p} \operatorname{Cov}\left(\Delta a_{\ell}, \Delta a_{k}\right) t^{\ell+k} .
\end{aligned}
$$

Direct substitution for the bias and covariance of the parameters gives complicated expressions. Without loss of generality, we again use the data centering trick, that is, the estimate is assumed to be computed at $t=0$. This simplification must also be taken care of in implementation, that is, if the signal estimate is desired at a point $t$, then the windowed data about that point must be translated to lie about the origin. The simplified expressions for bias and variance are applicable only under this realignment of data during computation of the estimator.

At $t=0$, we have that $\operatorname{Bias}(\widehat{s}(0))=$ $\mathcal{E}\left\{\Delta a_{0}\right\}, \operatorname{Var}\{\widehat{s}(0)\}=\operatorname{Var}\left\{\Delta a_{0}\right\} \quad$ Substituting for $\mathcal{E}\left\{\Delta a_{0}\right\}$ and $\operatorname{Var}\left\{\Delta a_{0}\right\}$, we have,

$$
\begin{aligned}
\operatorname{Bias}(\widehat{s}(0)) & =\frac{(-1)^{p+1} s^{p+1}(0)}{(p+1) !} \frac{\int \beta^{p+1} h(\beta) \mathrm{d} \beta}{\int h(\beta) \mathrm{d} \beta}, \text { and } \operatorname{Var}(\widehat{s}(0)) \\
& =\sigma_{w}^{2} \frac{\int h^{2}(\beta) \mathrm{d} \beta}{\left[\int h(\beta) \mathrm{d} \beta\right]^{2}} .
\end{aligned}
$$

\section{References}

[1] Rudin W 1973 Principles of mathematical analysis. 3rd edn, McGraw-Hill

[2] Fan J and Gijbels I 1996 Local polynomial modeling and its applications. Chapman and Hall, London

[3] Hardle W 1990 Applied Nonparametric Regression. Cambridge University Press, Cambridge

[4] Savitzky A and Golay MJE 1964 Smoothing and differentiation of data by simplified least-squares procedures. Anal. Chem. 36(8): 1627-1639

[5] Schafer RW 2011 What is a Savitzky-Golay filter? IEEE Signal Process. Mag. 28(4): 111-117

[6] Krishnan SR and Seelamantula CS 2013 On the selection of optimum Savitzky-Golay filters. IEEE Trans. Signal Process. 61(2): 380-391

[7] Kay SM 1988 Modern spectral estimation: Theory and applications. Prentice Hall, Englewood Cliffs, New Jersey

[8] Katkovnik V and Stankovic L 1998 Instantaneous frequency estimation using the wigner distribution with varying and data-driven window length. IEEE Trans. Signal Process. 46: 2315-2325 
[9] Stankovic L and Katkovnik V 1998 Algorithm for the instantaneous frequency estimation using time frequency distributions with adaptive window length. IEEE Signal Process. Lett. 5(9): 224-227

[10] Zhang ZG, Hung YS and Chan SC 2011 Local polynomial modeling of time-varying autoregressive models with application to time-frequency analysis of event-related EEG. IEEE Trans. Biomed. Eng. 58(3): 557-566

[11] Yao K and Thomas JB 1967 On some stability and interpolatory properties of nonuniform sampling expansions. IEEE Trans. Circuit. Theory CT-14: 404-408

[12] Jerri AJ 1977 The Shannon sampling theorem and its various extensions and applications: A tutorial review. Proc. IEEE 65: $1565-1596$

[13] Eldar Y and Oppenheim A V 2000 Filterbank reconstruction of bandlimited signals from nonuniform and generalized samples. IEEE Trans. Signal Process. 48: 2864-2875

[14] Prendergast RS, Levy BC and Hurst PJ 2004 Reconstruction of band-limited periodic nonuniformly sampled signals through multirate filter banks. IEEE Trans. Circuits Syst. - I 51(8): 1612-1622

[15] Margolis E and Eldar YC 2008 Nonuniform sampling of periodic bandlimited signals. IEEE Trans. Signal Process. 56(7): 2728-2745

[16] Strohmer T and Tanner J 2007 Fast reconstruction algorithms for periodic nonuniform sampling with applications to timeinterleaved ADCs. In: Proceedings of IEEE International Conference Acoustics Speech and Signal Processesing. Vol. 3, pp. 881-884.

[17] Divi V and Wornell G 2008 Bandlimited signal reconstruction from noisy periodic nonuniform samples in time-interleaved adcs, In: Proceedings of IEEE International Conference Acoustics Speech and Signal Processing, pp. 3721-3724
[18] Choi H and Baraniuk R 1999 Interpolation and denoising of nonuniformly sampled data using wavelet-domain processing. In: Proceedings of IEEE International Conference Acoustics Speech and Signal Processesing. pp. 1645-1648

[19] Laakso TI, Tarczynksi A, Murphy NP and Valimaki V 2000 Polynomial filtering approach to reconstruction and noise reduction of nonuniformly sampled signals. Signal Process. pp. 567-575

[20] Rauth M and Strohmer T 1998 Smooth approximation of potential fields from noisy scattered data. Geophysics 63(1): 85-94

[21] Strohmer T 1993 Efficient methods for digital signal and image reconstruction from nonuniform samples, $\mathrm{PhD}$ thesis, University of Vienna

[22] Stankovic L 2004 Performance analysis of the adaptive algorithm for bias-to-variance trade off. IEEE Trans. Signal Process. 52(5): 1228-1234

[23] Stankovic LJ and Stankovic S 1995 An analysis of instantaneous frequency estimation using time-frequency distributions - generalized Wigner distribution. IEEE Trans. Signal Process. 43(2): 549-552

[24] Chandra Sekhar S and Sreenivas TV 2003 Adaptive spectrogram vs. adaptive pseudo-Wigner-Ville distribution for instantaneous frequency estimation. Signal Process. 83(7): 1529-1543

[25] Donoho DL 1995 Denoising by soft thresholding. IEEE Trans. Info. Theory 41(3): 613-627

[26] Ristic B and Boashash B 1998 Comments on "the CramerRao lower bound for signals with constant amplitude and polynomial phase". IEEE Trans. Signal Process. 46(6): 1708-1709 